\title{
Whole Heliosphere Interval: Overview of JD16
}

\author{
David F. Webb ${ }^{1},{ }^{2}$ Sarah E. Gibson ${ }^{3}$ \\ and Barbara J. Thompson ${ }^{4}$ \\ ${ }^{1}$ ISR, Boston College, Institute for Scientific Research, 140 Commonwealth Ave., Chestnut \\ Hill, MA 02467 \\ ${ }^{2}$ Also at: Air Force Research Laboratory, Hanscom AFB, MA, 01731-3010, USA \\ email: david.webb@hanscom.af.mil \\ ${ }^{3} \mathrm{HAO} / \mathrm{NCAR}$, P.O. Box 3000, Boulder, CO 80301-3000, USA \\ email: sgibson@ucar.edu \\ ${ }^{4}$ NASA/Goddard Space Flight Center, Greenbelt, MD 20771, USA \\ email: barbara.j.thompson@nasa.gov
}

\begin{abstract}
The Whole Heliosphere Interval is an international observing and modeling effort to characterize the three-dimensional interconnected solar-heliospheric-planetary system, i.e., the "heliophysical" system. WHI was part of the International Heliophysical Year, on the 50th anniversary of the International Geophysical Year, and benefited from hundreds of observatories and instruments participating in IHY activities. WHI describes the 3-D heliosphere originating from solar Carrington Rotation 2068, March 20-April 16, 2008. The focus of IAU JD16 was on analyses of observations obtained during WHI, and simulations and modeling involving those data and that period. Consideration of the WHI interval in the context of surrounding solar rotations and/or compared to last solar minimum was also encouraged. Our goal was to identify connections and commonalities between the various regions of the heliosphere.
\end{abstract}

Keywords. Sun: magnetic fields, Sun: coronal mass ejections (CMEs), interplanetary (IP) medium, (Sun:) solar-terrestrial relations

\section{Characterizing the 3-D Heliosphere}

JD16 began with the introductory talk of Thompson et al. (2009), presented by S. Gibson, which overviewed the science and status of the Whole Heliosphere Interval (WHI) effort. The overarching science goals for WHI are 1) to characterize the three-dimensional solar minimum heliosphere and 2) to connect the origins and effects of solar structure and activity through the solar wind to the Earth and other planetary systems. The challenges of incorporating a vast array of data, Sun to Earth, and comparing these to three-dimensional models, both local and global, were discussed in this talk (see figure 1) and also in the poster of Schroeder et al. (2009). A Sun-to-Earth mapping of WHI structures during Carrington Rotation (CR) 2068, both observed and modeled, was also presented in the poster of Gibson et al. (2009c).

The invited talk of Bisi et al. (2009a) (this issue), presented by B. Jackson, (see also Bisi et al. (2009b)) summarized heliospheric observations, including interplanetary scintillation (IPS) observations from the Solar-Terrestrial Environment Laboratory (STELab) in Japan, as well as in-situ solar wind observations obtained by the STEREO, ACE, and Wind spacecraft for the WHI period. The global structure of the inner heliosphere was reconstructed tomographically from IPS observations, and compared favorably to insitu measurements. The invited talk of Manoharan (2009) (this issue) described another 


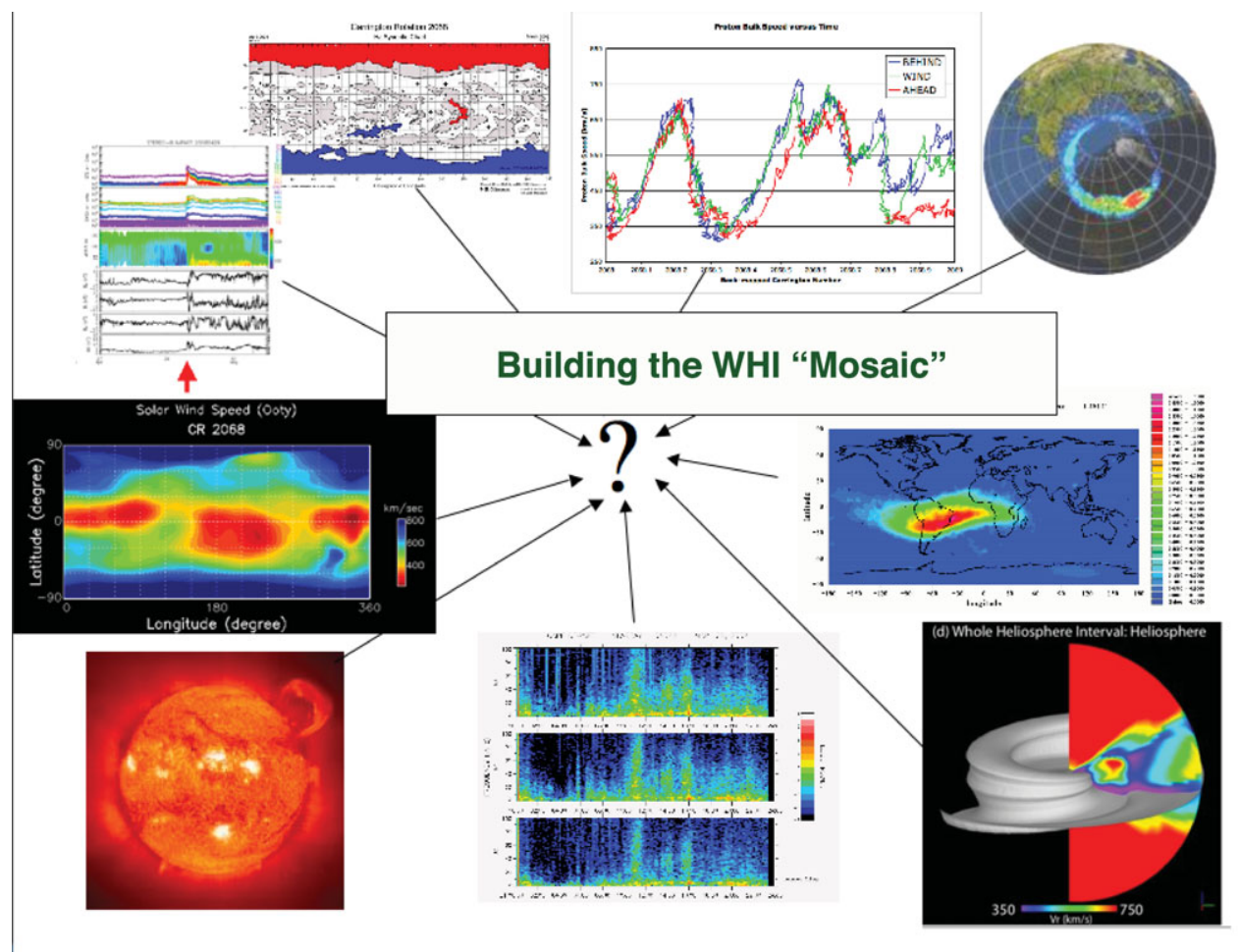

Figure 1. A fundamental and necessary challenge of WHI is combining data and models from a wide variety of sources. WHI participants have analyzed data from the solar subsurface, through the heliosphere, to the Earth's atmosphere. The above figure shows examples of the many types of data and model simulations comprising the WHI "Mosaic." Clockwise from upper right: Auroral UV emission, projected onto a map of Earth's north pole (Polar UVI), Energetic particle flux above Earth in the inner Van Allen belt (Jason-1 Doris), Modeled 3-D solar wind speed for CR 2068 (MAS Model), PC1 wave power spectrogram from Atlantic Search Coil Magnetometer at Cape Dorset, Antarctica, SOHO/EIT image of the solar chromosphere showing a giant erupting prominence, Solar wind speed for CR 2068 from IPS measurements (Ooty Radio Telescope), density, electron flux and magnetic field in a CME from IMPACT on STEREO-B, Magnetic polarity and coronal hole boundary map for CR 2068 (HelioSynoptics), and solar wind speed observed at STEREO-A, Wind, and STEREO-B, ballistically projected back to solar "origination" date.

observational analysis of the heliosphere using IPS data from the Ooty Radio Telescope, and compared the WHI period and the current solar minimum to the solar minimum of 1996. This analysis found that the band of low-speed solar wind along the equatorial belt was considerably wider during WHI than in the past minimum. The invited talk of Dal Lago et al. (2009) (this issue) presented cosmic ray observations from the Global Muon Detector Network (GMDN). They found that the cosmic ray gradient observed during WHI was consistent with the location of the heliospheric current sheet (HCS).

The invited talk of Riley et al. (2009) (this issue) presented a 3-D model of the solar corona and inner heliosphere during WHI. The MHD model included energy transport processes, estimated plasma temperature and density in the corona, and generally provided a global framework for the Sun and heliosphere during WHI. Model results were consistent with the IPS tomographic reconstruction of a wider band of variable and lowspeed wind around the equator for WHI, in particular as compared to the 1996 Whole Sun Month (WSM) period. Moreover, the model showed that there was a significant 
The "Active Side" of the WHI Sun: March 29, 2008
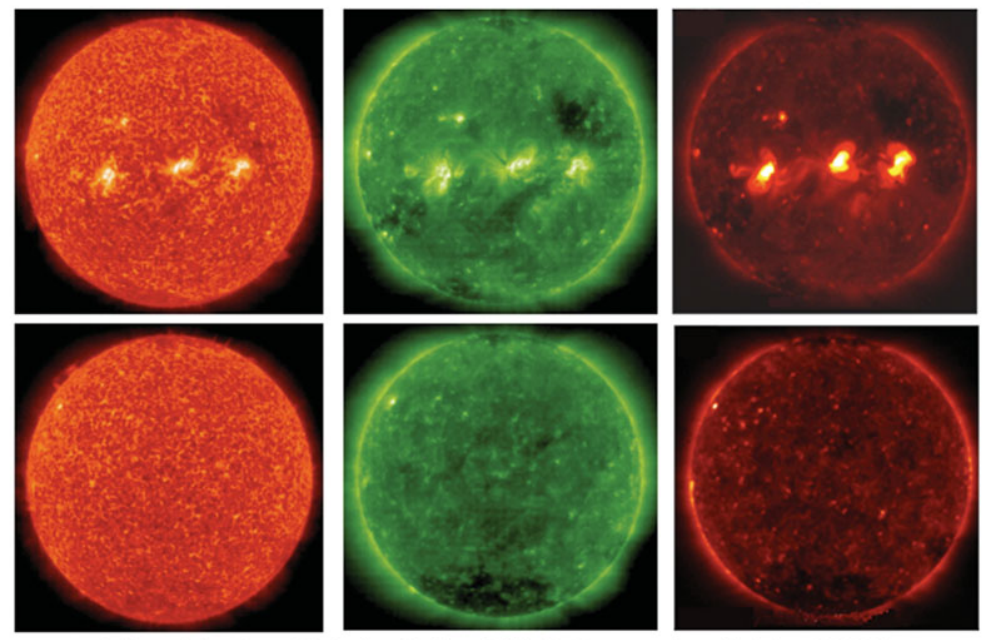

The "Quiet Side" of the WHI Sun: April 11, 2008

Figure 2. SOHO EIT 304A, 195A, and Hinode XRT soft X-ray observations of the corona during the "quiet" side of WHI (bottom) and the active side of WHI (top).

source of high-speed solar wind streams during WHI, as opposed to WSM, discussed further below.

The current solar minimum has been longer and quieter than any other observed during the Space Age, as described in an invited talk by D. Hassler. Our hope was that WHI would provide observations characteristic of this deep solar minimum, but also that the Sun would have sufficient structure and activity to make connections from Sun to Earth clear and compelling. Our hopes were realized.

For approximately the later half of the WHI period, the Sun presented a sunspot-free, deep solar minimum face (figure 2) (bottom panels). During this "quiet" side of WHI, on April 14, 2008, a NASA sounding rocket was launched from White Sands Missile Range in New Mexico, USA with a prototype EUV Variability Experiment (EVE), as described in the poster by Chamberlin \& Woods (2009), presented by M. Haberreiter (see also Woods et al. (2009) and Chamberlin et al. (2009)). The main purpose of this rocket flight was to provide the fifth underflight calibration for the Solar EUV Experiment (SEE). However, of great importance to WHI and solar minimum science, this rocket flight also provided the first observations of the solar EUV irradiance at high spectral resolution during solar cycle minimum conditions. The higher spectral resolution measurements will help resolve outstanding concerns about the previous solar soft X-ray irradiance results made with broadband photometers shortward of $27 \mathrm{~nm}$, and the measurements provide the most accurate reference for the 2008-2009 solar cycle minimum.

Figure 2 (top panels) shows soft X-ray and extreme ultraviolet observations of the Sun during the first half of WHI on CR 2068. Large, low-latitude coronal holes and solar active regions (ARs) are apparent. These departures from the quiet Sun seen later in the rotation led to both eruptive activity and solar wind structure that inspired many of the presentations at JD16.

Petrie \& Amari (2009) presented models of the magnetic structure of the three ARs visible during WHI, i.e., NOAA 10987, 10988 and 10989, day by day as they traversed the solar disk. Daily observations from the the SOLIS Vector Spectro-magnetograph were 
used as boundary conditions on the nonlinear force-free magnetic field code XTRAPOL. Physical quantities such as free magnetic energy and helicity were computed with reference to the potential field of these ARs, and properties of model fields were compared with virial estimates based on the magnetograms. The interconnectivity of the the three regions was also considered in the context of global potential field models of CR 2068 .

The invited talk of Leamon \& McIntosh (2009a) (also Leamon \& McIntosh (2009b)) demonstrated the influence of both coronal holes and ARs on the distribution of open magnetic field lines. In particular, they presented a new method of visualizing the solar photospheric magnetic field based on the "Magnetic Range of Influence" (MRoI). The MRoI is a simple realization of the magnetic environment, reflecting the distance required to balance the integrated magnetic field contained in any magnetogram pixel. Maps of MRoI allow for easy visual inspection of where sub-terrestrial field lines in a Potential Field Source Surface (PFSS) model connect to the photosphere and thus the source of Earth-directed solar wind. High MRoI can result from either a flux concentration (e.g., an AR), or from a large predominantly unipolar region (e.g., a coronal hole) which lead to very different wind conditions at $1 \mathrm{AU}$. In this study, the MRoIs during both the WHI and WSM periods were determined. In particular, the WHI period differed greatly from the WSM period in that the large, low-latitude coronal holes dominated the Sun-Earth connectivity and were the source of fast solar wind that drove responses at Earth.

\section{Connecting Origins and Effects of WHI Structure and Activity}

High-speed streams emerging from low-latitude coronal holes were a key characteristic of the WHI interval, and for the months both before and after WHI. Although sunspot numbers were lower than the previous minimum, long-lived regions of open magnetic flux at low latitudes meant that the Earth was periodically driven by these regions as they appeared rotation after rotation. The invited talk of Dal Lago et al. (2009) (this issue) described two streams during WHI that created corotating interaction regions (CIRs), with highly fluctuating magnetic fields, that had geomagnetic impact. Dense structures associated with these CIRs, as well as other transient structures, were reconstructed in the inner heliosphere from Solar Mass Ejection Imager (SMEI) data, as described in the talk of Jackson et al. (2009). This dense material was then compared with the CIR velocity patterns from the aforementioned IPS data. The invited talk of Maris \& Maris (2009) (this issue) described the extent that the wind streams of WHI and surrounding rotations drove geomagnetic storms, and compared the high-speed streams of WHI and surrounding rotations to those seen during WSM. The talk of Gibson et al. (2009a) also compared the origins and impacts of high-speed streams during WHI to those of WSM, noting that the outer radiation belt observed by the GOES satellites during WHI were at levels more than $3 \times$ that of WSM, and that both the radiation belts and aurorae exhibited the periodicities of the solar wind due to the recurring high speed streams.

Most of the $\sim 60 \mathrm{CMEs}$ or other transients during WHI occurred during the first, active half of the interval (see figure 2). A working group was organized to study these transients, both individual events and how the events fit into the global and evolutionary pattern of the solar magnetic field. The overarching goal was to trace the effects of solar structure and activity through the solar wind to Earth, other planets and spacecraft.

The discussion of activity was keynoted in an invited talk by Sterling (2009) (this issue) who gave an overview of CME observations and models. He described his examination of the lower solar atmosphere at EUV wavelengths during WHI, searching for indications of eruptive events. He used EIT data from SOHO and EUVI data from the two STEREO 
spacecraft, and found that, of the 3 main ARs during WHI, the most potential-like region 10989 was also the most dynamic, also confirmed by Petrie \& Amari (2009).

An overview of specific projects involving data on activity during the WHI interval was given by Webb et al. (2009). He discussed the origins and early development of the CMEs, including data on their associated ARs and filaments and prominences, the use of multi-spectral data to study the propagation of the transients over large distances and in 3 domensions, and studies of the events in the context of the global magnetic field and the heliospheric current sheet. He discussed the use of helioseismology data from SOHO MDI (D. Haber) to determine flows as a function of depth in two areas of activity during CRs 2068 and 2069. Haber found a possible vortex area before the April 5-9 series of events, and flows before the April 26 event. These periods are being studied globally and compared with changes in $\mathrm{H}$-alpha synoptic charts (with P. McIntosh).

Cremades et al. (2009) discussed a study to characterize all the CMEs that originated during WHI, including their source regions and ensuing IP structures, aiming toward a global description of their role in determining heliospheric conditions. They also included narrower ejective features such as "jets". Data from the STEREO and SOHO spacecraft allow the study of 3-D properties of the CMEs and multi-wavelength images from these missions and from ground-based observatories help characterize the CME source regions. The association of the Earth-directed CMEs with IP CMEs (ICMEs), including magnetic clouds, was assessed using data from the ACE spacecraft at L1. These results during WHI were then compared with those from the WSM in 1996. They found for WHI vs WSM that there were more ejecta on all size scales, less ambiguity in source identification, ejecta occurred at all position angles with jets at polar latitudes, and the solar wind was less oriented along the Parker spiral direction. Models of magnetic clouds observed during this solar cycle minimum were also discussed in the poster by Vandas \& Romashets (2009).

Lisnichenko \& Podladchikova (2009) described results during WHI using the continuous EUV imaging of the solar corona by the STEREO/SECCHI/EUVI and SOHO/EIT telescopes to detect key surface signatures of CMEs. These include the global EIT waves and coronal "dimming" regions. They compared the results using both visual examination of images and with a detection algorithm called NEMO (Novel EIT wave Machine Observing). They produced a catalog of WHI EIT wave and dimming events and discussed their morphology. Visually they found 10 large-scale EUV events associated with CMEs. Using SOHO data NEMO autonomously detected 40 Events, and using STEREO data detected 42 events. The WHI events had substantially smaller geometrical sizes than events observed in 1997-2005.

Energetic, fast CMEs with shocks (e.g., Type II bursts) occurred during WHI on March 25 and just after WHI on April 26, 2008 and were associated with arcades, coronal dimmings and EUV waves. The March $25 \mathrm{CME}$ was a classic limb CME, and the April 26 CME originated on the disk and appeared as an ICME at STEREO-B. The March 25 event was discussed in the talks by Gopaslwamy et al. (2009a) (see also Gopalswamy et al. (2009b)) and Webb et al. (2009). The STEREO spacecraft were separated by about $50^{\circ}$, so the CME was a disk event from STEREO-B and a limb event for STEREO-A. One of the important aspects of this CME is that it was well observed by the STEREO/SECCHI inner coronagraph (COR1) when the metric type II burst was in progress, so they were able to obtain the shock height with respect to the CME.

Other details of the April 26 event were described by Jackson et al. (2009) and Webb et al. (2009). A brief IP type II suggested that this event had the lowest starting frequency ever observed, with implications for the medium through which shock propagates. At STEREO-B the Fe charge state data suggested that there was a CIR-type interface with bidirectional streaming indicating possible ejecta. SMEI density movies confirm 
this showing that the CME broke through the pre-existing CIR with its core passing just east and north of STEREO-B with some plasma hitting Earth.

The three WHI ARs had several transients starting on April 5 until their west limb passges several days later. A west-limb CME on April 9 was asociated with 10989 when it was over the limb and a filament to its east. The CME was slow with an erupting prominence and post-CME current sheet observed by Hinode XRT. Del Zanna (2009) reported on EUV spectral observations performed by the SOHO Coronal Diagnostic Spectrometer during the filament eruption, together with broad-band imaging observations from X-rays (Hinode/XRT) to the EUV (STEREO, TRACE). The EUV spectra were used to explain the observed signatures in the images. Surprisingly, little evidence of heating was found in these observations, including in the X-ray emission. Webb et al. (2009) also presented analyses of the filament, CME and current sheet from XRT observations by S. Savage and D. McKenzie, and Hinode, STEREO, and SOHO observations by Landi et al. (2009).

Rapid pulsations in solar flares at sub- $\mathrm{THz}$, mid-IR and $\mathrm{GHz}$ ranges observed by the Solar Submillimeter-wave Telescope (SST) in Argentina were discussed in the talk by Kaufmann et al. (2009) and the poster by Cassiano et al. (2009). Rapid radio pulses in solar bursts have been known for many decades and are common to all events when observed with enough time resolution and sensitivity. These new observations combined with other imaging and spectral data can be used to better understand the processes in ARs that lead to flares and CMEs.

Besides the modeling of the WHI ARs by Petrie \& Amari (2009) discussed earlier, two other talks dealt with modeling issues. In the talk of Stepanov, Tsap and Kopylova (2009), given by Y. Tsap, they used the ballooning instability driven by kink oscillations to model the formation of the cusp-shaped structure atop a coronal loop that can form during a CME as a result of magnetic reconnection. They compared their results to microwave and hard X-ray observations from an event on April 15, 2002. In an invited talk, Kusano et al. (2009) described data-driven simulations of Sun-Earth connection events which goals are to understand the physical mechanisms of the missing links in the Sun-Earth system, and to develop a physics-based model applicable for the prediction of the onset of solar eruptions.

\section{Space Weather and Future Work}

Kusano et al. (2009) also discussed the use of end-to-end modeling to predict the influence of solar eruptions on the terrestrial environment, or space weather. Their group is developing a new simulation model of the heliosphere, consisting of several sub-models of solar ARs, the corona, IP space, Earths magnetosphere, and particle acceleration. The model was applied to the geoeffective event on Dec. 13-15, 2006. The simulation was driven by Hinode vector magnetograms of the magnetic field of the flaring AR, and solar wind observations including IPS data. Future work includes better coupling of the sub-models, developing a high-accuracy interpolation between different metrics, improving the SEP model, and combining MHD and hybrid simulations. Although there were no Hinode vector field data during WHI, it was suggested that ground-based SOLIS magnetograms might be used to drive the simulation for WHI events.

In an invited talk, Raulin et al. (2009a) (this issue) discussed the future of the IHY and its campaigns, of which WHI was one. The IHY program took place over the 5 year period 2004-2008 and was a major international effort that involved the deployment of new instrumentation, new observations from the ground and in space, and a strong education component. Under the United Nations, instrument arrays were deployed to 
provide global measurements of heliophysical phenomena. The UN is planning to continue this effort aimed at understanding the impacts of Space Weather on Earth and the near-Earth environment as a new program, the International Space Weather Initiative (ISWI). In a poster Raulin et al. (2009b) described one of the UN IHY programs called SAVNET. This is the South America VLF Network which consists of seven tracking receivers spread over Latin America. The SAVNET array is monitoring anomalies of subionospheric propagating VLF waves that reveal changes of the electrical properties of the low ionospheric D- and E-regions. Results were shown of SAVNET's capability to monitor solar activity on short, minutes to hours, and long, solar cycle, time scales.

Schuch et al. (2009) and Kemmerich et al. (2009) discussed the use of ground-based muon detectors to measure the high energy galactic cosmic ray intensity and its modulation by the magnetic field in transients such as CMEs and CIRs. The limited observations during WHI were discussed. Schuch et al. (2009) proposed to combine an existing 6country network of global muon multi-directional detectors telescopes, called the GMDN, with STEREO observations to develop a new method to track in 3-D solar-heliospheric structures. The reduction in cosmic ray counts at Earth's surface caused by ICMEs and their shocks traveling in IP space can be detected as much as ten hours in advance with the GMDN, permitting more accurate and reliable Space Weather forecasts.

Finally, Gavryuseva (2009) discussed a study of the relationships between the solar and IP magnetic fields, the solar wind characteristics near Earth and geomagnetic perturbations using Wilcox Solar Observatory observations of the large-scale solar magnetic field and OMNI solar wind data over 30 years and 3 solar cycles. The goal was to search for variations of the latitudinal and longitudinal structure of the photospheric field to make long-term predictions of solar activity leading to space weather events. The temporal dependence of the differential rotational rate was determined as a function of latitude and a quasi-stable longitudinal structure persisted over the 30-year period.

\section{Acknowledgements}

We thank the following who were either members of the SOC for JD16 or reviewed the papers in this section: D. Banerjee, M. Bisi, A. Breen, H. Cremades, N. Crosby, J. Davila, R. Forsyth, A. Galvin, K. Georgieva, J. Kozyra, I. Mann, P. Manoharan, G. Petrie, G. Poletto, K. Shibata and R. Stamper. The NASA STEREO mission consists of 4 primary suites of remote sensing and in situ instruments on two spacecraft and comprises multiple instruments developed by many worldwide groups. Hinode is a Japanese mission developed and launched by ISAS/JAXA, with NAOJ as domestic partner and NASA and STFC (UK) as international partners, and in co-operation with ESA and NSC (Norway). $\mathrm{SOHO}$ is a project of international collaboration between ESA and NASA. SMEI is a collaborative project of the US Air Force Research Laboratory, NASA, the University of California at San Diego, the University of Birmingham, UK, Boston College, and Boston University.

\section{References}

Bisi, M. M., Jackson, B. V., Clover, J. M., Hick, P. P., Buffington, A., \& Tokumaru, M. 2009a, Highlights of Astronomy, XXVIIth IAU General Assembly, August 2009, Ian F. Corbett, ed. (this issue) 15

Bisi, M. M., Jackson, B. V., Buffington, A., Clover, J. M., Hick, P. P., \& Tokumaru, M. 2009b, Solar Phys. 256, 201

Cassiano, M. M. et al. 2009, XXVII IAU General Assembly Abstract Book 391

Chamberlin, P. C. \& Woods, T. N. 2009, XXVII IAU General Assembly Abstract Book 387 
Chamberlin, P. C., Woods, T. N., Crotser, D. A., Eparvier, F. G., Hock, R. A., \& Woodraska, D. L. 2009, Geophys. Res. Lett., 36, 5, CiteID L05102

Cremades, H., S. Dasso, \& C. H. Mandrini, 2009 XXVII IAU General Assembly Abstract Book 387

Dal Lago, A. et al. 2009, Highlights of Astronomy, XXVIIth IAU General Assembly, August 2009, Ian F. Corbett, ed. (this issue) 15

Del Zanna, G. 2009, XXVII IAU General Assembly Abstract Book 386

Gavryuseva, E. A. 2009, XXVII IAU General Assembly Abstract Book 387

Gibson, S. E., Kozyra, J. U., De Toma, G., Emery, B. A., Onsager, T., \& Thompson, B. J. 2009a, XXVII IAU General Assembly Abstract Book 386

Gibson, S. E., Kozyra, J. U., De Toma, G., Emery, B. A., Onsager, T., \& Thompson, B. J. 2009b, J. Geophys. Res. 114, A09105, doi:10.1029/2009JA014342

Gibson, S. E. et al. 2009c, XXVII IAU General Assembly Abstract Book 392

Gopalswamy, N. et al. 2009a, XXVII IAU General Assembly Abstract Book 390

Gopalswamy, N. et al. 2009b, Solar Phys, 259: 227254

Jackson, B. V. et al. 2009, XXVII IAU General Assembly Abstract Book 386

Kaufmann, P., C. G. Gimnez de Castro, E. Correia, J. E. R. Costa, J.-P. Raulin, \& A. S. Vlio 2009, XXVII IAU General Assembly Abstract Book 391

Kemmerich, N. et al. 2009, XXVII IAU General Assembly Abstract Book 392

Kusano, K. et al. 2009, XXVII IAU General Assembly Abstract Book 390

Landi, E., J. C. Raymond, M. P. Miralles, \& H. Hara 2009, Astrophys. J., submitted

Leamon, R. J. \& McIntosh, S. W. 2009a, XXVII IAU General Assembly Abstract Book 387

Leamon, R. J. \& McIntosh, S. W. 2009b, Astrophys. J. Lett 697, L28

Lisnichenko, P. and O. Podladchikova 2009, XXVII IAU General Assembly Abstract Book 387

Manoharan, P. K. 2009, Highlights of Astronomy, XXVIIth IAU General Assembly, August 2009, Ian F. Corbett, ed. (this issue) 15

Maris, G. \& Maris, O. 2009, Highlights of Astronomy, XXVIIth IAU General Assembly, August 2009, Ian F. Corbett, ed. (this issue) 15

Petrie, G. \& Amari, T. 2009, XXVII IAU General Assembly Abstract Book 386

Raulin, J.-P., J. M. Davila, T. Bogdan, K. Yumoto, \& J. Leibacher 2009a, XXVII IAU General Assembly, August 2009, Ian F. Corbett, ed. (this issue) 15

Raulin, J.-P., F. Bertoni, \& H. Rivero 2009b, XXVII IAU General Assembly Abstract Book 389

Riley, P., Linker, J. A., \& Mikic, Z. 2009, Highlights of Astronomy, XXVIIth IAU General Assembly, August 2009, Ian F. Corbett, ed. (this issue) 15

Schroeder, P., Thompson, B., \& Gibson, S. 2009, XXVII IAU General Assembly Abstract Book 392

Schuch, N. J. et al. 2009, XXVII IAU General Assembly Abstract Book 388

Stepanov, A. V., Y. T. Tsap, \& Y. G. Kopylova 2009, XXVII IAU General Assembly Abstract Book 387

Sterling, A. C. 2009, XXVII IAU General Assembly, August 2009, Ian F. Corbett, ed. (this issue) 15

Thompson, B. J. et al. 2009, XXVII IAU General Assembly Abstract Book 390

Vandas, M. \& E. Romashets 2009, XXVII IAU General Assembly Abstract Book 391

Webb, D. F. et al. 2009, XXVII IAU General Assembly Abstract Book 389

Woods, T. N. et al. 2009, Geophys. Res. Lett., 36, 1, CiteID L01101 


\section{JD16 ORGANIZING COMMITTEE}

Barbara J. Thompson (Chair, USA)

David F. Webb (Co-chair, USA)

Andrew R. Breen (UK)

Norma B. Crosby (Belgium)

Antoinette B. Galvin (USA)

Janet U. Kozyra (USA)

Giannina Poletto (Italy)

Richard Stamper (UK)
Sarah E. Gibson (Co-chair, USA)

Dipankar P.K. Banerjee (India)

Hebe Cremades (Argentina)

Robert J. Forsyth (UK)

Katya Y. Georgieva (Bulgaria)

Ian R. Mann (Canada)

Kazunari Shibata (Japan)

\section{Acknowledgements}

This joint discussion was coordinated through IAU Division II (Sun and Heliosphere) and sponsored and supported by IAU Division X (Radio Astronomy), and by IAU Commissions 10 (Solar Activity), 12 (Solar Radiation and Structure) and 49 (Interplanetary Plasma and Heliosphere).

Funding support by the International Astronomical Union, International Heliophysical Year and

National Aeronautics and Space Administration is gratefully acknowledged. 